Original Article

\title{
A PROSPECTIVE OBSERVATIONAL STUDY ON THE ASSESSMENT OF POTENTIAL DRUG-DRUG INTERACTIONS AND MANAGEMENT OF POST-COMPLICATIONS IN STROKE PATIENTS AT A TERTIARY CARE TEACHING HOSPITAL
}

\author{
*MAHE NAAZ SULTANA, SABA YOUNUS QURESHI, IQRA FATIMA, WAJIDA JABEEN, MOHAMMED OBAID
}

Department of Pharmacy Practice, MESCO College of Pharmacy, Hyderabad 500006, Telangana, India

Email: mahenaaz99@gmail.com

Received: 24 Jun 2021, Revised and Accepted: 11 Sep 2021

\section{ABSTRACT}

Objective: The objective of the study is to determine the occurrence, frequency, and severity of significant drug-drug interactions in stroke patients, with an emphasis on post complications and their symptomatic management.

Methods: A prospective observational study was carried out for 6 mo at Osmania General Hospital, a Tertiary Care Teaching Hospital. The prescriptions taken by stroke patients throughout their hospital stay were analyzed for possible interactions using-Micromedex-2 (Thomson Reuters) × 2.0.

Results: A total of 130 prescriptions was analyzed in the study in which 141 drug-drug interactions were found with a male predominance of 85.4\%. The majority of interactions were of moderate severity (74.46\%) and pharmacokinetics (67.37\%) in nature. Among the clinical consequences, decreased atorvastatin efficacy $(49.10 \%)$ was found to be the highest. The class of drugs most commonly involved in drug-drug interactions was found to be anti-epileptics and statins. $60.15 \%$ of the study population were identified with one complication, among which seizures account for $15 \%$ of patients. All the complications allied with the condition were treated with drug classes in the study population.

Conclusion: This study highlighted the exigency for screening prescriptions of cerebrovascular accident patients for potential drug-drug interactions (pDDIs). Brain edema and seizure were the most accountable complications identified in the study population. The early detection of these problems can improve stroke outcomes and can reduce the mortality or disability rate.

Keywords: Stroke, Potential drug-drug interactions, Interaction severity, Micromedex, Post-complications

(C) 2021 The Authors. Published by Innovare Academic Sciences Pvt Ltd. This is an open access article under the CC BY license (https://creativecommons.org/licenses/by/4.0/) DOI: https://dx.doi.org/10.22159/ijpps.2021v13i11.42527. Journal homepage: https://innovareacademics.in/journals/index.php/ijpps.

\section{INTRODUCTION}

Innocuous use of modern and conventional medicines is a striving objective for all of us. Medicines have brought massive benefits, but no medicine is $100 \%$ safe and sound for all people. While some medicines can gravely injure or even kill, chiefly most of them have beneficial effects for the people even while they may cause intermittent minor harm such as headache, rash, or tiredness [1].

Drug therapy is an essential part of disease management. The use of multiple drugs which are required either to manage a single disease or co-morbidities can cause harmful interactions between them [2]. Drug-related problems such as adverse drug reactions, drug-drug interactions, idiosyncratic reactions, and hypersensitivity were major challenges in clinical practice [3]. Potential DDIs are observed to be one of the most frequently appearing challenges that may amend the drug pharmacokinetics and pharmacodynamics, thus altering the overall therapeutic response [4].

An interaction is said to occur when the effects of one drug are altered by the co-administration of another drug, herbal medicine, food, drink, or other environmental chemical agents. The net effect of the combination may manifest as an additive or enhanced effect of one or more drugs, antagonism of the effect of one or more drugs, or any other alteration in the effect of one or more drugs [5].

Certain conditions such as multiple disorders, chronic diseases, and polypharmacy may enhance the risk of potential DDIs. Drug therapies in critically ill patients are often complicated by the altered physiology and co-existence of multiple co-morbidities that warrant poly-pharmacy. The management of the disease typically requires the use of more than one drug. When patients have multiple symptoms, it becomes decisive to prescribe copious drugs. Drugdrug interactions usually occur amidst drugs with a low therapeutic index, with a small difference between their therapeutic and toxic or lethal doses [6]. This means the slightest change in the dosage of a drug can produce dangerous and harmful events.
According to the World Health Organization (WHO), stroke (Apoplexy, the word used by Hippocrates) is a condition "characterized by rapidly developing clinical symptoms and/or signs of focal, and at times global, loss of cerebral function, with symptoms lasting more than $24 \mathrm{~h}$ leading to death with no apparent cause other than that of vascular origin" [7]. There are two main types of cerebrovascular accidents or stroke: Ischemic stroke and hemorrhagic stroke. In both cases, part of the brain is deprived of blood and oxygen, causing the brain cells to die [8].

Worldwide, cerebrovascular diseases are accountable for 6.15 million deaths [9]. Stroke is the second leading cause of death worldwide and can lead to stern, long-term disability. In addition to neurological deficits, diverse medical complications can arise following a stroke [10]. Furthermore, complications from stroke meddle with early rehabilitation, therapy and prevent recovery [11].

Thus, the study aims to determine the occurrence, frequency, and severity of significant drug-drug interactions in stroke patients, with an emphasis on post complications and their symptomatic management.

\section{MATERIALS AND METHODS}

Study design and population

A prospective observational study was carried out for a period of 6 mo (November 2019-April 2020) in the General medicine department and Neurology IPD of Osmania General Hospital in Hyderabad (Telangana), which is an 1100 bedded tertiary care hospital with male and female departments, the largest government clinical setting in southern India. Ethical approval was obtained from the institutional ethics committee (MCP/IEC/PD/PR/45) before the initiation of the study according to the institution's regulations. Prescriptions of 130 stroke patients admitted consecutively to inpatient wards of a tertiary care hospital were analyzed during this study. Prescriptions with two or more drugs prescribed throughout the hospitalization were only preferred for 
the study. The study population comprised of patients above18 $\mathrm{y}$ and who had a length of stay $>24 \mathrm{~h}$. Stroke patients with other comorbidities were included and patients unwilling to participate, visit the outpatient department, pregnant and lactating women were excluded from the study.

\section{Data collection}

Demographic information (age and gender), number of drugs prescribed, habit history (alcohol and tobacco), length of hospital stays, general examination with lab parameters, main diagnosis, medication chart, and follow-up details were obtained from the patient's case record form. Laboratory data reports and clinical records of the patient's previous medical history were collected from the patient/caretaker through an interview. Certain demographic characteristics were considered to identify the prognosticators of DDIs, such as patient characteristics [length of hospital stay], and the number of drugs per prescription. The medications taken by the stroke patients during their hospital stay were analyzed for possible DDIs via an electronic database-Micromedex x 2.0. Available data on prescriptions included are Name, strength, frequency, and quantity of medications dispensed. The data obtained was used to categorize interactions based on the mechanism as pharmacokinetic or pharmacodynamics. The interactions were classified into mild, moderate, and severe according to the severity scale.

\section{Drug-drug interaction checker}

Micromedex electronic database tool was used to identify and analyze the pattern of DDIs [12]. Micromedex contains a separate section on DDIs known as the Drug-REAX System. On entering the drugs one by one, the program lists the possible DDIs and categorizes DDIs according to their interaction effect, severity (contraindicated, serious, moderate, mild, and unknown), onset (rapid, delayed, and unspecified), management, documentation status (excellent, good, fair, poor, unlikely, and unknown), and literature reports.

\section{Statistical analysis}

The data subjected to statistical analysis in the present study included the number of drugs prescribed, length of hospital stay, and number of DDIs. The data were analyzed for descriptive statistics and further with an independent t-test for the test of significance at the $5 \%$ level using Microsoft Excel 2010.

\section{RESULTS}

A total of 130 stroke prescriptions was included in the study, and among them, hemorrhagic stroke was 71 (54.61\%), followed by ischemic stroke 59 (45.38\%). The average stay in the hospital was $13 \mathrm{~d}$. The analysis of stroke prescriptions allowed us to identify 141 interactions among 86 patients, with an average number of drugs taken of 7.15. All the patient prescriptions were analyzed during the study period and it was found that 53 (37.6\%) patients were confirmed with a minimum of one DDI. A substantial proportion of patients with drug-drug interactions are occupied by males 111 $(85.4 \%)$. Patients with an age group of $41-60$ y had $82(58.15 \%)$ DDIs and were followed by other age groups. The patient characteristics are summarized in (table 1).

Table 1: Demographic profile of stroke patients

\begin{tabular}{llc}
\hline Parameter & Total no. of patients & Percentage \\
\hline Gender wise distribution & 111 & $85.38 \%$ \\
Male & 19 & $14.61 \%$ \\
Female & 22 & $16.92 \%$ \\
Age-wise distribution & 67 & $51.53 \%$ \\
$20-40$ Y & 41 & $31.53 \%$ \\
$41-60$ Y & & $23.68 \%$ \\
$61-80$ Y & 63 & $29.32 \%$ \\
Social habits & 78 & $1.87 \%$ \\
Smoker & 05 & $96.16 \%$ \\
Alcoholic & 125 & $3.84 \%$ \\
Betel Nut & 05 & $45.38 \%$ \\
No. of drugs in prescription & & \\
$>5$ Drugs & 59 & $54.61 \%$ \\
< Drugs & 51 & \\
Type of stroke & 5 & \\
Ischemic & & \\
Hemorrhagic & & \\
\hline
\end{tabular}

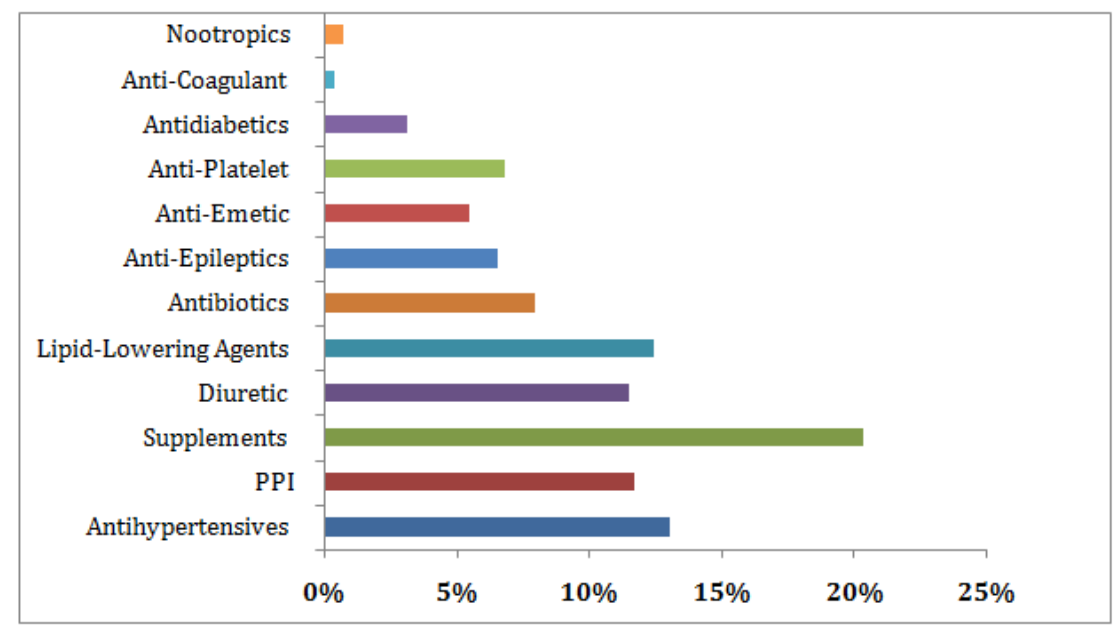

Fig. 1: Drug utilization pattern 
The most common drug classes (fig. 1) prescribed to the stroke patients were supplemented, anti-hypertensives, and lipid-lowering agents followed by diuretic and PPIs. The incidence rate of DDIs in the present study was anticipated to be $86 \%$.

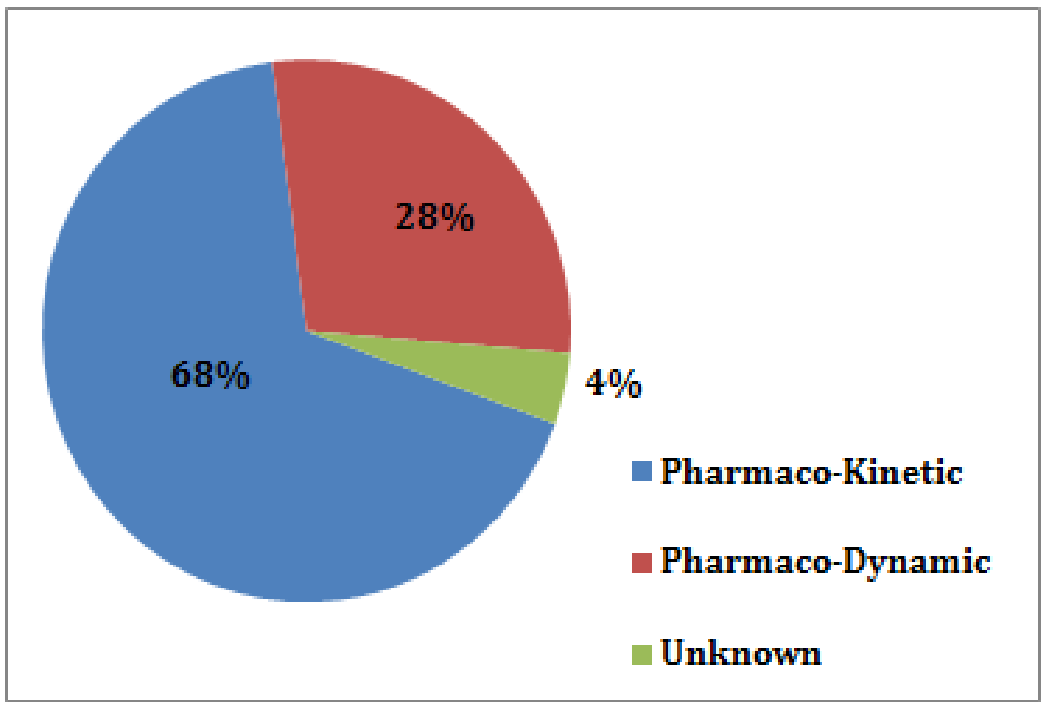

Fig. 2: Type of interactions found from database

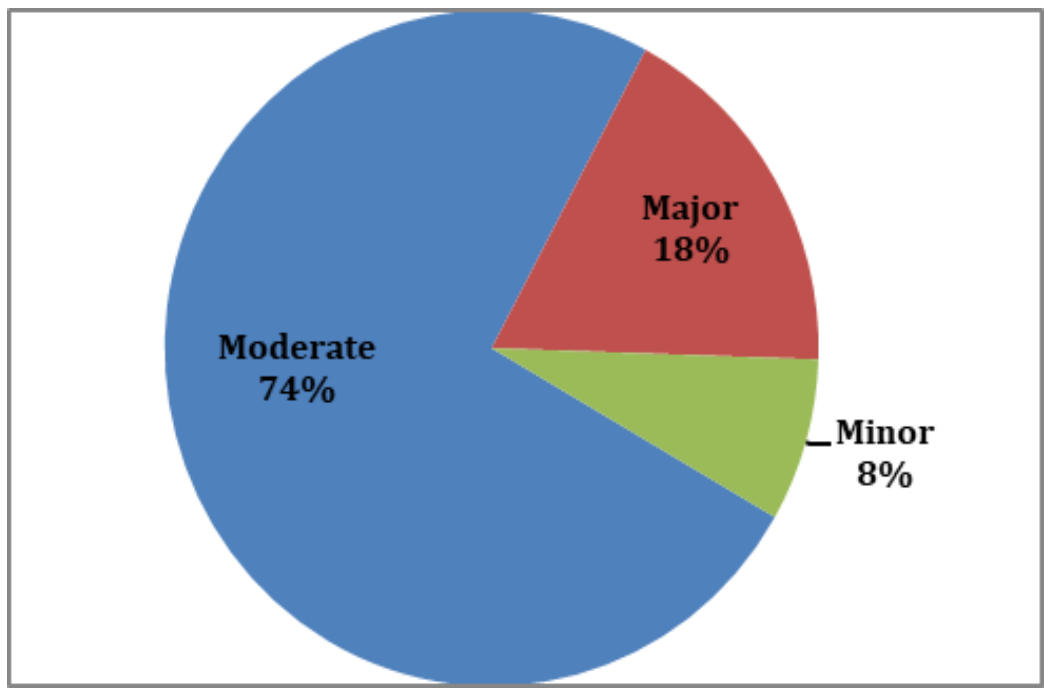

Fig. 3: Type of pDDIs based on the severity scale

This study revealed there were 105 moderate, 25 major, and 11 minor interactions (fig. 3). Among these, pharmacokinetic interactions were 95 and the pharmacodynamics interactions were 40 followed by unknown 6 in (fig. 2).

Table 2: List of commonly occurring drug-drug interactions

\begin{tabular}{ll}
\hline Interacting pair & Clinical consequences \\
\hline Aspirin/Clopidogrel & Increased Risk of Bleeding \\
Amlodipine/Clopidogrel & Increased Risk of Thrombotic Events \\
Phenytoin/Calcium Carbonate & Decreased Exposure of Phenytoin \\
Aspirin/Metformin & Increased Risk of Hypoglycemia \\
Phenytoin/Atorvastatin & Decreased Atorvastatin Plasma Concentration and Efficacy \\
Aspirin/Calcium Carbonate & Decreased Salicylates Effectiveness \\
Enalapril/Aspirin & Decreased Effectiveness of Enalapril \\
Phenytoin/Acetaminophen & Increased Risk of Hepatotoxicity \\
\hline
\end{tabular}

In our study, the above-mentioned list of drug-drug interactions-aspirin/clopidogrel, amlodipine/clopidogrel, aspirin/metformin, and phenytoin/calcium carbonate were of major severity; whereas the interacting rest pairs were of moderate severity 


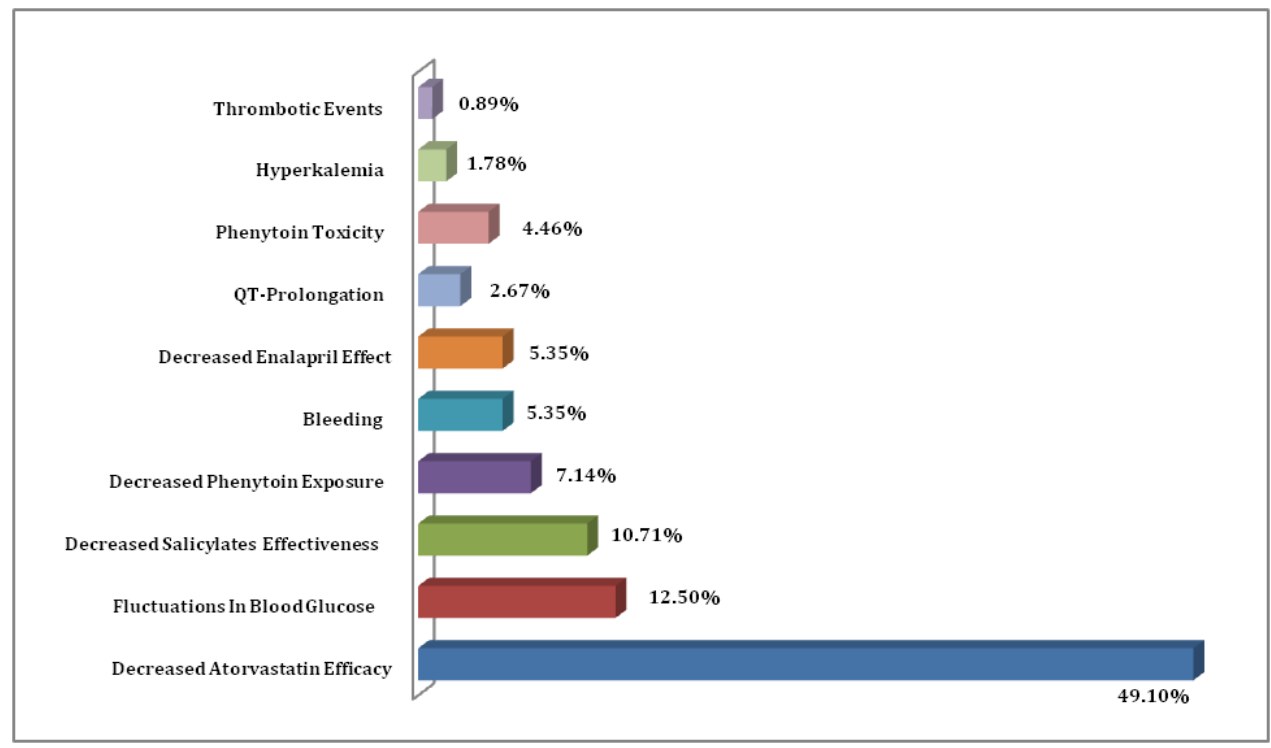

Fig. 4: Bar graph showing various clinical consequences occurring due to DDI's, Among the various clinical consequences, decreased atorvastatin efficacy $(49.10 \%)$ was found to be the highest

Table 3: Management options required for DDI's

\begin{tabular}{llc}
\hline Management & Number of DDIs & Percentage \\
\hline Dose Adjustment & 69 & $44.23 \%$ \\
Monitor for Biochemical Parameters & 23 & $14.74 \%$ \\
Dose-Titration & 22 & $14.10 \%$ \\
Monitor for Drug Levels & 09 & $5.76 \%$ \\
Avoid Combination & 07 & $4.48 \%$ \\
Monitor for Sign and Symptoms & 07 & $4.48 \%$ \\
No Management Required & 06 & $3.84 \%$ \\
Risk-Benefit Analysis & 06 & $3.84 \%$ \\
Alternative Medication & 05 & $3.20 \%$ \\
Monitor for Electrolyte Levels & 02 & $1.28 \%$ \\
\hline
\end{tabular}

It was noted that the most common management plan found for most of the DDIs was dose adjustment accounted for the highest percentage $(44.23 \%)$

Table 4: Type of complication and their percentage

\begin{tabular}{lll}
\hline Complications & No. of patients & Percentage \\
\hline Edema & 105 & $65.62 \%$ \\
Seizures & 26 & $16.25 \%$ \\
Aspiration Pneumonitis & 18 & $11.25 \%$ \\
Urinary Tract Infection & 11 & $6.87 \%$ \\
\hline
\end{tabular}

It was noted that $60.15 \%$ of the study population were identified with one complication, among which seizures account for $15 \%$ of patients. $68 \%$ of patients developed brain edema, incorrigible by CT scans in 2-5 d of their disease onset. The individual percentages of patients with complications are summarized in (table 4).

Table 5: Drug utilization in the therapy of post-stroke complications

\begin{tabular}{lll}
\hline Complications & A drug used & No. of patients \\
\hline Edema & Mannitol & 105 \\
Seizures & Phenytoin & 26 \\
Aspiration Pneumonitis & Amoxicillin & 18 \\
Urinary-Tract Infection & Levofloxacin & 11 \\
\hline
\end{tabular}

All the complications allied with the condition were treated with drug classes in the study population.

Edema, due to the pathophysiological changes of ischemia, was controlled by using an osmotic diuretic (mannitol) in 105 patients $(65.62 \%)$. The different complications and their respective management options are listed in (table 5).
Association between the risk factors and incidence of DDI's in stroke patients

Length of hospital stay and number of medicines prescribed were assessed to determine their connection with the likelihood of incidence of DDIs. The data analysis revealed a negative correlation between these factors and the incidence of DDI's. 
Table 6: Descriptive statistics and correlation between length of hospital stay and number of DDI's

\begin{tabular}{|c|c|c|c|c|c|c|c|}
\hline Parameters & $(\mathrm{N})$ & Mean & Median & SD & Standard error & Sample variance & Confidence level (95\%) \\
\hline Length of hospital stay & 130 & 13.5 & 13.5 & 3.605 & 1.040 & 13 & 2.290 \\
\hline No. of DDI's & 130 & 11.75 & 9 & 10.549 & 3.045 & 111.2 & 6.702 \\
\hline Parameters & \multicolumn{2}{|c|}{ P-value (one tail) } & \multicolumn{2}{|c|}{ P-value (two tail) } & R-value & \multicolumn{2}{|c|}{ Linear regression equation } \\
\hline $\begin{array}{l}\text { Length of hospital stay } \\
\text { No. of DDI's }\end{array}$ & \multicolumn{2}{|c|}{0.29604} & \multicolumn{2}{|c|}{0.59208} & -0.237 & \multicolumn{2}{|c|}{$\begin{array}{l}y=-0.4273 x+18.1 \\
R^{2}=0.0176\end{array}$} \\
\hline
\end{tabular}

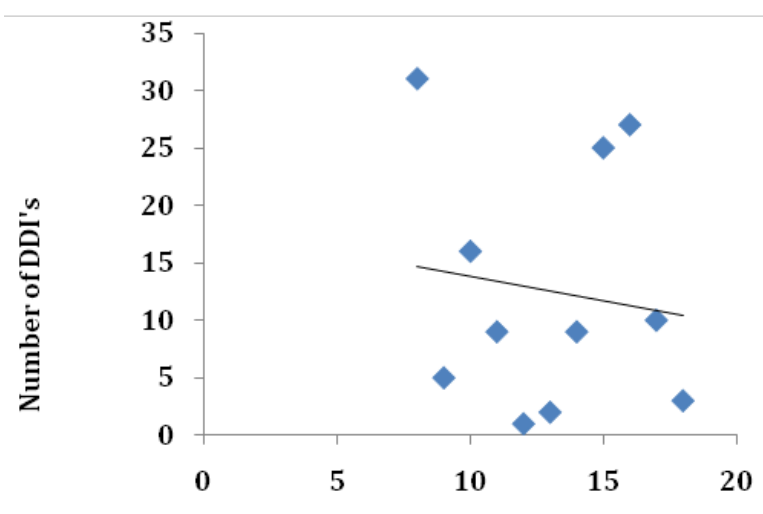

Length of hospital stay

Fig. 5: Linear regression between the length of hospital stay and number of DDI, Therefore, there is a negative correlation between the length of hospital stay and the number of drug interactions

Table 7: Descriptive statistics and correlation between no. of medicines prescribed and number of DDI's

\begin{tabular}{|c|c|c|c|c|c|c|c|}
\hline Parameters & $\mathbf{N}$ & Mean & Median & SD & Standard error & Sample variance & Confidence level (95\%) \\
\hline No. of drugs prescribed & 130 & 8 & 8 & 2.738 & 0.912 & 7.5 & 2.105 \\
\hline No. of DDI's & 130 & 15.66 & 18 & 11.045 & 3.681 & 122 & 8.490 \\
\hline Parameters & \multicolumn{2}{|c|}{ P-value (one tail) } & \multicolumn{2}{|c|}{ P-value (two tail) } & R-value & \multicolumn{2}{|c|}{ Linear regression equation } \\
\hline $\begin{array}{l}\text { No. of drugs prescribed } \\
\text { No. of DDI's }\end{array}$ & \multicolumn{2}{|c|}{0.0301} & \multicolumn{2}{|c|}{0.0603} & -0.264 & \multicolumn{2}{|c|}{$\begin{array}{l}y=-1.0667 x+24.2 \\
R^{2}=0.0699\end{array}$} \\
\hline
\end{tabular}

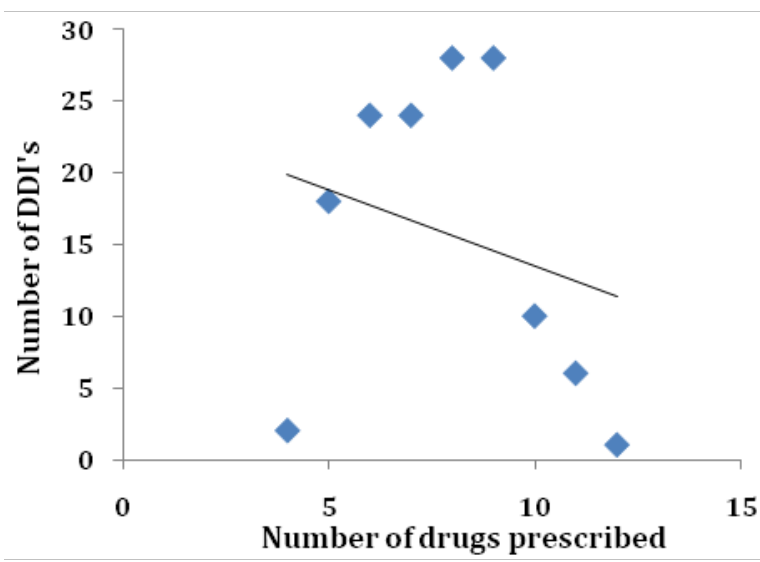

Fig. 8: Linear regression between the number of medicines and number of DDI's, Therefore, there is a negative correlation between the number of medicines and the number of drug interactions

\section{DISCUSSION}

The present study revealed the overall incidence of pDDIs among 130 stroke patients at Osmania General Hospital. Out of 130 stroke patients, 111 (85.38\%) were males and 19 (14.61\%) were females. The occurrence of stroke was more in men than women. This was similar to the study conducted by N. Anitha et al. $(63.8 \%$ were male and $36.2 \%$ were female) (2020) [18].
Drug-drug interactions (DDI's) are the major concern for patients, and this risk increases as larger numbers of medications are frequently used to handle intricate conditions. DDI has always been a subject of concern in the effective management of a patient's ill health.

The average number of drugs per prescription was 7.15. The finding was found to be similar to the study findings conducted by 
Thammisetty D. P et al., (7.2) (2021) [19]. The overall incidence rate of pDDIs in the present study was found to be $86 \%$ which is more than the study conducted by Virendra KP et al., (30.67\%) (2011) [20].

A sum of 141 drug-drug interactions was found in 130 patients with a male predominance of $85.38 \%$. The predominance was similar to the studies conducted by Virendra KP et al. (males 66\%) and Sushmita et al., (males-61\%) [20]. The most common drug classes involved in DDIs were the anti-epileptics, stations, antiplatelets, and antihypertensives as shown in fig. 1. Among this phenytoin, atorvastatin, aspirin, clopidogrel, amlodipine, furosemide, and enalapril were the drugs that caused common clinically significant drug interactions.

On analyzing the mechanism of drug interaction, pharmacokinetics type of interactions (67.37\%) was found to be in greater number compared to pharmacodynamic drug interactions $(28.36 \%)$ followed by the interactions of unknown mechanism (4.25\%). This finding was found to be similar to the study findings conducted. On account of severity assessment of the reactions, the majority of the reactions were categorized as moderate $(74.46 \%)$, followed by major severity $(17.73 \%)$. The finding was similar to a study conducted.

A strong inverse correlation was found between the length of hospital stay and DDIs ( $r=-0.237$, p-value is $=0.29604$ ). The similar significant negative correlation was also found between the number of medicines prescribed and DDI's ( $r=-0.264$, $p$-value $=0.0301$ ).

The most common management plan found for most of the DDIs was Dose Adjustment (44.23\%). These results were following the observation of studies conducted. The suggested action to be taken in most cases was monitoring biochemical parameters, signs and symptoms,+-electrolyte levels.

Our study revealed the various medical and neurological complications which are allied to stroke following the acute attack. The identification of such complications in the initial days of effect helps to perceive the early preventability. In our study, a high incidence of patients was with one complication i.e., edema (67\%). The result was in congruence with the observation of earlier reported studies. In the current study, all the identified complications were managed as per their standard guidelines for symptomatic relief.

\section{CONCLUSION}

From a pharmacological perspective, drug-drug interactions are real with indecisive clinical implications. Our study highlighted the bodice, which was high in stroke patients above $40 \mathrm{y}$. The majority of interactions were pharmacokinetic, having moderate severity. The significant proportions of patients with DDIs were occupied by males followed by the females.

In this study, pDDIs mainly occurred between anti-epileptics, stations antiplatelet, and antihypertensives. A clinical pharmacist plays a vital role in identifying the timely drug interactions and reporting them to the physician which may be helpful to prevent potentially detrimental drug interactions. Long-term complications are frequent in stroke survivors. Special care should be provided to patients with risk factors for post-stroke medical complications, including old age, poor initial motor function, poor swallowing function, and pre-existing morbidities. The accurate identification of the risk factors allied with the stroke patients prior; help us to bring down the cost burden along with improvement in the quality of life effectively.

\section{LIMITATION OF STUDY}

The sample size of the study was relatively small due to the short duration of the study. The current study was attributable only to a single hospital. The study was conducted without any intervention component. Further prospective studies are needed for the observation, identification, and management of DDIs among stroke patients.

\section{ACKNOWLEDGMENT}

We wish to thank the management of the MESCO College of Pharmacy, the principal, vice-principal for their support and cooperation in the execution of this research work. We also duly express our thanks to the institution-affiliated teaching hospital administration for their cooperation.

\section{FUNDING}

Nil

\section{AUTHORS CONTRIBUTIONS}

All authors have contributed equally.

\section{CONFLICTS OF INTERESTS}

Declared none

\section{REFERENCES}

1. Uppsala Monitoring Centre (UMC). World Health Organization Collaborating Centre for International Drug Monitoring; 2020.

2. Abideen S, Vivekanandan K, Mishra P. Assessment of the prevalence of potential drug-drug interactions in medical intensive care unit of a tertiary care hospital in India. Asian J Pharm Clin Res. 2015;8:125-313.

3. Krähenbühl-Melcher A, Schlienger R, Lampert M, Haschke $M$, Drewe J, Krähenbühl S. Drug-related problems in hospitals: a review of the recent literature. Drug Saf. 2007;30:379-407. doi: 10.2165/00002018-200730050-00003, PMID: 17472418

4. Karen Baxter PCL. Stockley's drug interactions. 9th ed. London, UK: Pharmaceutical Press; 2010.

5. Walker R, Whittlesea C. Textbook of clinical pharmacy and therapeutics. 5th ed. 50. New York: Churchill Livingstone; 2012.

6. Mounica B. A prospective study on drug-drug interactions in the medication charts in general medicine wards, in a tertiary Care Hospital. Guntur, Andhra Pradesh, and the clinical pharmacists role. Int J Biol Pharm Res. 2014:374-7.

7. Ingeman A, Andersen G, Hundborg HH, Johnsen SP. Medical complications in patients with stroke: data validity in a stroke registry and a hospital discharge registry. Clin Epidemiol. 2010;2:5-13. doi: 10.2147/clep.s8908, PMID 20865097.

8. Albers GW, Caplan LR, Easton JD, Fayad PB, Mohr JP, Saver JL, Sherman DG, TIA Working Group. Transient ischemic attack-proposal for a new definition. N Engl J Med. 2002;347(21):17136. doi: 10.1056/NEJMsb020987, PMID 12444191.

9. WHO. The top 10 causes of death online address; 2011. Available from: http://www.who.int/mediacentre/factsheets/ fs310/en/index. [Last accessed on 01 Jul 2015].

10. Doshi VS, Say JH, Young SH, Doraisamy P. Complications in stroke patients: a study carried out at the rehabilitation medicine service, changi general hospital. Med J. 2003;44:643-52.

11. Dromerick A, Reding M. Medical and neurological complications during inpatient stroke rehabilitation. Stroke. 1994;25(2):35861. doi: 10.1161/01.str.25.2.358, PMID 8303745.

12. DRUGDEX® System [database on CD-ROM]. Version 5.1. Greenwood Village, CO: Thomson Reuters. Healthcare; 2013.

13. Anitha N. To estimate the incidence of potential drug-drug interaction in stroke patients admitted in a tertiary Care Hospital. Int J Curr Pharm Res. 2020;12:48-52. doi: 10.22159/ijcpr.2020v12i2.37487.

14. Thammisetty DP, Ranganayakulu D, Nayakanti D. Drug-related problems and its prescribing indicators in stroke patients: a prospective observational study. Asian J Pharm Clin Res. 2021;14:41-4. doi: 10.22159/ajpcr.2021.v14i3.40529.

15. Patel VK, Acharya LD, Rajakannan T, Surulivelrajan M, Guddattu V, Padmakumar R. Potential drug interactions in patients admitted to cardiology wards of a South Indian teaching hospital. Australas Med J. 2011;4(1):9-14. doi: 10.4066/AMJ.2011.450, PMID 23393498.

16. Sharma S, Chhetri HP, Alam K. A study of potential drug-drug interactions among hospitalized cardiac patients in a teaching hospital in Western Nepal. Indian J Pharmacol. 2014;46(2):1526. doi: 10.4103/0253-7613.129303, PMID 24741184.

17. Sivva D, Mateti UV, Neerati VM, Thiruthopu NS, Martha S. Assessment of drug-drug interactions in hypertensive patients at a super speciality hospital. Avicenna J Med. 2015;5(2):29-35. doi: 10.4103/2231-0770.154194, PMID 25878964 . 
18. Mateti UV, Rajakannan T, Nekkanti H, Rajesh V, Mallaysamy SR, Ramachandran P. Drug-drug interactions in hospitalized cardiac patients. J Young Pharm. 2011;3(4):329-33. doi: 10.4103/0975-1483.90246, PMID 22224041.

19. Kulkarni V, Bora SS, Sirisha S, Saji M, Sundaran S. A study on drug-drug interactions through prescription analysis in a South Indian teaching hospital. Ther Adv Drug Saf.
2013;4(4):141-6. doi: 10.1177/2042098613490009, PMID 25114777.

20. Johnston KC, Li JY, Lyden PD, Hanson SK, Feasby TE, Adams RJ, Faught RE, Haley EC Medical and neurological complications of ischemic stroke: experience from the RANTTAS trial. RANTTAS Investigators. Stroke. 1998;29(2):447-53. doi: 10.1161/ 01.str.29.2.447. PMID 9472888. 